\title{
A rare case of juvenile localised scleroderma with intra-oral and dental involvement
}

\author{
PENG WANG, WEI GUO and SHUTAI LIU \\ Department of Periodontology, Yantai Stomatological Hospital, Yantai, Shandong 264008, P.R. China
}

Received October 13, 2014; Accepted September 1, 2015

DOI: $10.3892 /$ etm.2015.2791

\begin{abstract}
Juvenile localised scleroderma is a rare childhood disorder of the immune system and connective tissue with unknown etiology. There are different types of localised scleroderma, including linear scleroderma (where the lesion appears as a line or streak) and plaque or circumscribed morphea (where the lesion appears as a roundish lesion). The present report describes the case of a 10-year-old girl, who presented with a history of linear scleroderma with nose, oral and dental involvement, and outlines the diagnosis of the case based on the clinical presentation, pathology, X-ray and cone beam computed tomography images. The treatment strategy that was selected for the patient is additionally discussed.
\end{abstract}

\section{Introduction}

Juvenile localised scleroderma (JLS), known as 'morphea' in the dermatological literature, refers to a variety of conditions characterised by skin thickening with increased collagen deposition (1). The connective tissue diseases that constiTtute JLS are rare, complex and have an unknown etiology. Localised plaques are typically benign or self-limiting; however, the condition may be cosmetically disfiguring and result in permanent alopecia. JLS includes five subtypes: Circumscribed morphoea, linear scleroderma, generalized morphoea, pansclerotic morphoea and the mixed subtype if a combination of two or more of the previous subtypes is present (2). Linear lesions may progress to invade the subcutaneous tissue, muscle and bone, leading to limb atrophy, contracture and limb length discrepancies, thus resulting in functional disabilities or severe cosmetic disfigurement. Early diagnosis is critical, as early therapeutic protocols can improve the patient's quality of life, slow disease progression and assist with improved disease management. To date, few reports

Correspondence to: Dr Shutai Liu, Department of Periodontology, Yantai Stomatological Hospital, 142 North Street, Yantai, Shandong 264008, P.R. China

E-mail: dentistliu@163.com

Key words: juvenile localised scleroderma, linear scleroderma, oral, dental, methotrexate describing cases of JLS are available in the literature, which reflects the rarity of the condition.

\section{Case report}

A 10-year-old girl presented with an 8-month history of white plaque at the skin of the left ala of the nose, with subsequent painless scarring extending onto the centre of the upper lip. A painless, pink, pitting plaque had initially appeared at the skin of the left ala of the nose 8 months previously. The plaque gradually expanded down through the columella nasi to the lip bead on the centre of the labrum. The skin of the plaque also gradually hardened and whitened. In the past 2 months, the maxillary anterior teeth had exhibited a gradual loosening. The patient was examined by doctors at local hospitals, but a firm diagnosis was not able to be provided. The patient apparently looked physically healthy, and no documented family history was available.

A facial examination revealed an ivory plaque extending from the skin of the left ala of nose to the lip bead on the centre of the upper lip. The lesion varied in width between 5 and $15 \mathrm{~mm}$. The left ala of the nose was slightly concave (Fig. 1A). An intra-oral examination revealed a white plaque extending from the centre of the upper lip mucosa to the groove of the oral vestibule, eventually extending to the labial gingivae attached to the left maxillary anterior tooth. The periodontal pocket depth of the round structure of the maxillary left central incisor and the mesial of the maxillary left lateral incisor was 13 and $12 \mathrm{~mm}$, respectively, which was deeper than that of the opposite tooth, which had normal bone structures. The labial gingiva of the maxillary left central incisor was eroded and shaped like a ' $\mathrm{V}$ '. The tooth mobility of the maxillary left central incisor was degree II (Fig. 1B) (3). An X-ray and cone beam computed tomography revealed alveolar bone resorption between the affected teeth (Fig. 2). Blood investigations, including a full blood count with differential, erythrocyte sedimentation rate, alkaline phosphatase and antinuclear, anti-Scl-70, anti-centromere and anti-ribonucleoprotein antibodies, were essentially normal, with the exception of a raised rheumatoid factor level (33.7 IU/ml). A biopsy from the labial gingivae in the region of the upper left central incisor was found to be an irregularly thickened squamous epithelium occupying the mucosal surface. The connective tissue collagen under the squamous cell layer exhibited hyaline degeneration and hardening (Fig. 3). Excess collagen can lead to fibrosis, which 

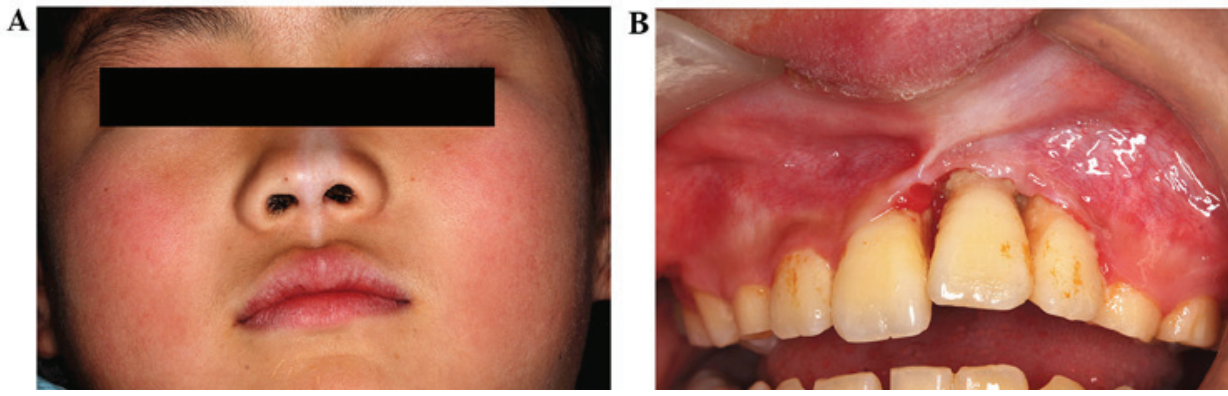

Figure 1. (A) An ivory plaque extended from the skin of the left ala of the nose to the lip bead on the centre of the upper lip. The lesion varied in width between 5 and $15 \mathrm{~mm}$. The left ala of the nose was slightly concave. (B) The labial gingiva of the maxillary left central incisor was eroded and shaped like a ' $\mathrm{V}$ '. The mobility of the maxillary left central incisor was degree II.

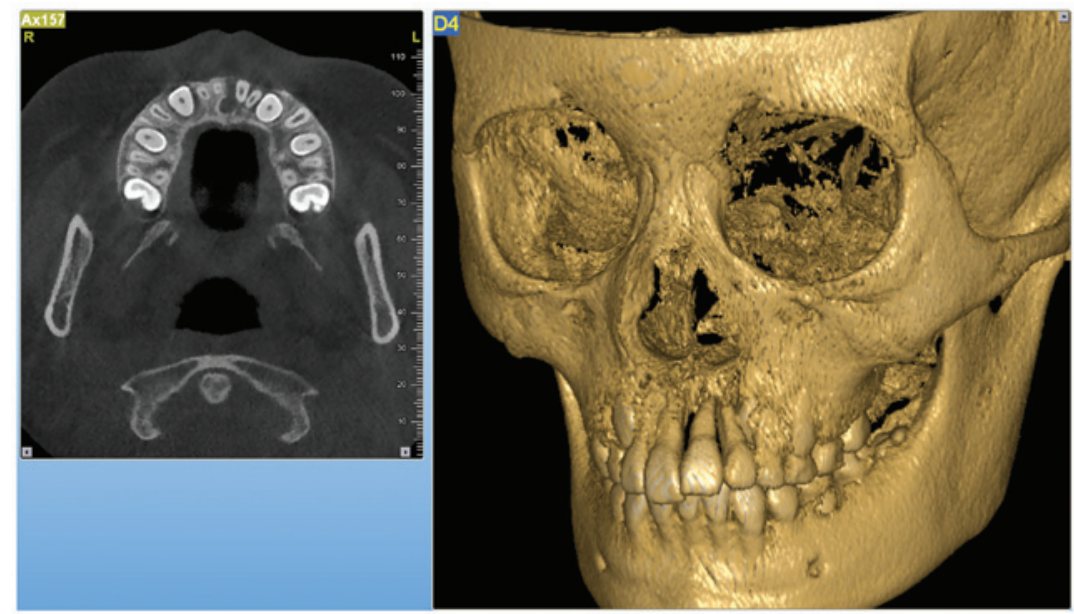

Figure 2. Cone beam computed tomography revealed alveolar bone resorption between the affected teeth (11,21 and 22).

A

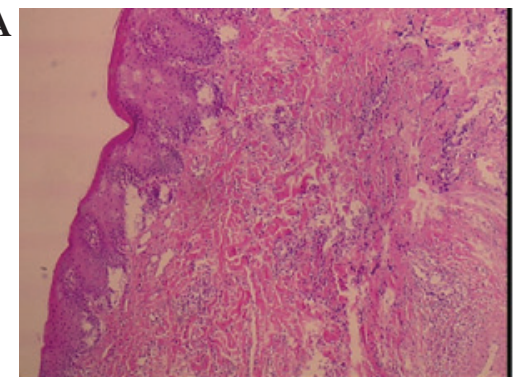

B

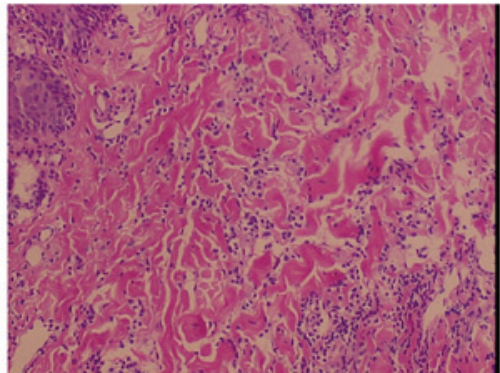

C

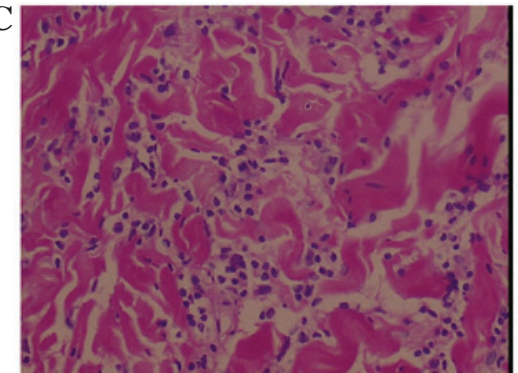

Figure 3. Biopsy from the labial gingivae in the region of the upper left central incisor showed irregularly thickened squamous epithelium occupying the mucosal surface. The connective tissue collagen that lay under the squamous cell layer was hyaline and exhibited degeneration and hardening. (A) Magnification, $\mathrm{x} 4$; (B) magnification, x10; (C) magnification, x20 (hematoxylin and eosin staining).

A

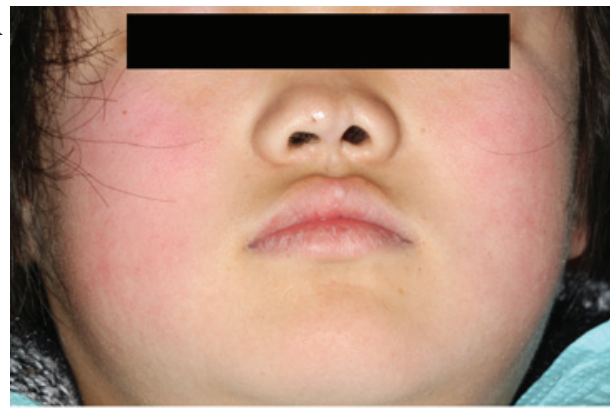

B

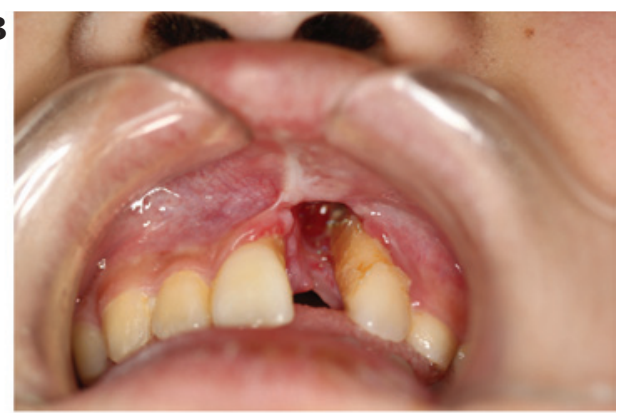

Figure 4. (A) Softening and significant lightening of the lesion of the face (nose and upper lip) was found at the 6-month follow-up, preventing the lesion from being detected due to its near-skin colour. (B) There was no healing in the mucosa or hard tissue of the mouth. Following the removal of the maxillary left central incisor due to mastication forces, the tooth extraction wound did not heal or close, even after 6 months. 
is similar to scarring. The patient was diagnosed with JLS, morphea-type, by a dermatologist at the Affiliated Hospital of Beijing University (Beijing, China) under remote consultation.

Glycyrrhizin capsules (Weifang Zhongshi Pharmaceutical Co., Ltd., Weifang, China), escin (Cesra Arzneimittel GmbH \& Co. KG, Baden-Baden, Germany) and total glucosides of paeony (Ningbo Lihua Pharmaceutical Co., Ltd., Ningbo, China) were prescribed to the patient in oral doses of $75 \mathrm{mg} /$ day, $300 \mathrm{mg} /$ day and $0.9 \mathrm{~g} / \mathrm{day}$, respectively, along with a topical application of tacrolimus and mucopolysaccharide polysulphate cream.

At the 6-month follow-up appointment, softening and significant lightening of the lesion of the face (nose and upper lip) were found (Fig. 4A), which prevented the lesion from being noticed due to its similarity in colour to the skin; however, no change in the healing of the mucosa or hard tissue of the mouth was apparent. The treatment plan included the extraction of the maxillary left central incisor, which was impossible to preserve; however, the parents initially refused the procedure due to the desire to preserve the tooth as long as possible. After 2 months, the maxillary left central incisor had to be pulled out due to mastication forces. Following the removal of the tooth, the extraction wound did not heal or close, even after 6 months (Fig. 4B).

\section{Discussion}

The more common subtypes of JLS include linear, plaque and mixed linear and plaque types. The exact cause of JLS is unknown, but a positive family history of rheumatic or autoimmune diseases and environmental factor triggers have been implicated (4). Similarly to other connective tissue diseases, JLS mainly involves females aged 7-10 years, which is consistent with previous studied $(5,6)$.

Linear lesions can invade the subcutaneous tissue, muscle and bone, resulting in tissue contracture and functional disabilities or severe cosmetic disfigurement (7). In the present case, the JLS had extended into the deep skin, involving the underlying muscle and alveolar bone. Due to the lack of understanding regarding JLS in clinicians who are not rheumatologists or dermatologists, this patient did not receive an exact diagnosis or effective therapy in the local hospital. The present case suggests that clinicians should improve their knowledge of JLS. In this case, histopathological examination was crucial for establishing a definite diagnosis.

There is no standard modality of treatment for JLS. Studies have shown that topical tacrolimus ointment can effectively reduce the skin thickening, pigment loss, hardness, erythema, capillary expansion and contraction $(8,9)$. Systemic medication with methotrexate (MTX) or combined treatment with MTX and glucocorticoids can be taken orally. Studies have shown that long-term MTX therapy is beneficial and well-tolerated for JLS $(10,11)$. In addition, photochemotherapy with ultraviolet A in combination with the photosensitiser psoralen has been successfully used to reduce skin fibrosis in localised scleroderma (morphea) (12). Glycyrrhizin and escin are well-known Chinese herbal medicines that have been used to inhibit inflammation, oxidation and immunoregulation. Research has shown that total glucosides of paeony can decrease the inflammatory reaction and enhance wound and ulcer healing (13). In the present case, oral glycyrrhizin capsules, escin and total glucosides of paeony were prescribed to the patient, along with a topical application of tacrolimus and mucopolysaccharide polysulphate cream. After 6 months of treatment, the fibrosis of the facial skin was alleviated significantly, and the colour and hardness of the local facial area were similar to those of the normal skin. Based on these results, it appears that the joint application of these three medications may be a valid alternative in the treatment of local scleroma. There was no change, however, in the healing of the mucosa and hard tissue of the mouth. Good oral health care is essential for patients with scleroderma, in order to prevent gingivitis and alveolar bone destruction. When a tooth is lost, referral to a restorative specialist is also required. A temporary partial denture should be used to maintain the clearance. Following stabilization, plastic surgery can help to reshape the face, and transplants using healthy tissue can restore the diseased tissue. Furthermore, loss of alveolar bone should be restored by means of bone graft, and a fixed prosthesis or an implant denture should be used to replace the missing incisor.

\section{Acknowledgements}

The present study was supported by the 'Taishan Scholars' Foundation (no. TSHW20120233) from Shandong Provincial Government. The authors would like to thank Professor Ping Ye (Institute of Dental Research, Centre for Oral Health, Westmead Hospital, Sydney, Australia) for the revision of the present manuscript.

\section{References}

1. Peterson LS, Nelson AM and Su WP: Classification of morphea (localized scleroderma). Mayo Clin Proc 70: 1068-1076, 1995.

2. Zulian F and Martini G: Preliminary classification criteria for juvenile systemic sclerosis. In: Zulian F and Ruperto N (eds): Proceedings of the II Workshop on nomenclature and diagnostic criteria for Juvenile Scleroderma Syndromes. Padua, pp5-16, 2005.

3. Newman MG, Takei H, Klokkevoid PR and Carranza FA (eds): Carranza's Clinical Periodontology. 11th edition. Elsevier Saunders, Philadelphia, PA, p347, 2012.

4. Browning JC: Pediatric morphea. Dermatol Clin 31: 229-237, 2013.

5. Murray KJ and Laxer RM: Scleroderma in children and adolescents. Rheum Dis Clin North Am 28: 603-624, 2002.

6. Uziel Y, Krafchik BR, Silverman ED, Thorner PS and Laxer RM: Localized scleroderma in childhood: A report of 30 cases. Semin Arthritis Rheum 23: 328-340, 1994.

7. Laxer RM and Zulian F: Localized scleroderma. Curr Opin Rheumatol 18: 606-613, 2006.

8. Cantisani C, Miraglia E, Richetta AG, Mattozzi C and Calvieri S: Generalized morphea successfully treated with tacrolimus $0.1 \%$ ointment. J Drugs Dermatol 12: 14-15, 2013.

9. Kroft EB, Groeneveld TJ, Seyger MM and de Jong EM: Efficacy of topical tacrolimus $0.1 \%$ in active plaque morphea: randomized, double-blind, emollient-controlled pilot study. Am J Clin Dermatol 10: 181-187, 2009.

10. Zulian F, Vallongo C, Patrizi A, Belloni-Fortina A, Cutrone M, Alessio M, Martino S, Gerloni V, Vittadello F and Martini G: A long-term follow-up study of methotrexate in juvenile localized scleroderma (morphea). J Am Acad Dermatol 67: 1151-1156, 2012.

11. Inamo $Y$ and Ochiai $T$ : Successful combination treatment of a patient with progressive juvenile localized scleroderma (morphea) using imatinib, corticosteroids, and methotrexate. Pediatr Dermatol 30: e191-e193, 2013.

12. Gambichler T, Terras S and Kreuter A: Treatment regimens, protocols, dosage and indications for UVA1 phototherapy: Facts and controversies. Clin Dermatol 31: 438-454, 2013.

13. Jia XY, Chang Y, Sun XJ, Wu HX, Wang C, Xu HM, Zhang L, Zhang LL, Zheng YQ, Song LH and Wei W: Total glucosides of paeony inhibit the proliferation of fibroblast-like synoviocytes through the regulation of $\mathrm{G}$ proteins in rats with collagen-induced arthritis. Int Immunopharmacol 18: 1-6, 2014. 